\title{
Density-Dependent Synthetic Gauge Fields Using Periodically Modulated Interactions
}

\author{
S. Greschner, ${ }^{1}$ G. Sun, ${ }^{1}$ D. Poletti, ${ }^{2}$ and L. Santos ${ }^{1}$ \\ ${ }^{1}$ Institut für Theoretische Physik, Leibniz Universität Hannover, Appelstrasse 2, DE-30167 Hannover, Germany \\ ${ }^{2}$ Engineering Product Development, Singapore University of Technology and Design, 20 Dover Drive, 138682 Singapore \\ and Merlion MajuLab, CNRS-UNS-NUS-NTU International Joint Research Unit, UMI 3654, Singapore \\ (Received 20 November 2013; revised manuscript received 5 September 2014; published 21 November 2014)
}

\begin{abstract}
We show that density-dependent synthetic gauge fields may be engineered by combining periodically modulated interactions and Raman-assisted hopping in spin-dependent optical lattices. These fields lead to a density-dependent shift of the momentum distribution, may induce superfluid-to-Mott insulator transitions, and strongly modify correlations in the superfluid regime. We show that the interplay between the created gauge field and the broken sublattice symmetry results, as well, in an intriguing behavior at vanishing interactions, characterized by the appearance of a fractional Mott insulator.
\end{abstract}

The emulation of synthetic electromagnetism in cold neutral gases has attracted major interest [1,2]. Artificial electric and magnetic fields have been induced using lasers [3-5]. Moreover, these setups may be extended to generate non-Abelian fields, and in particular, spin-orbit coupling [6-13]. Synthetic fields may be generated as well in optical lattices, and recent experiments have created artificial staggered [14-16] and uniform [17,18] magnetic fields. These fields are, however, static, as they are not influenced by the atoms.

The dynamical feedback between matter and gauge fields plays an important role in various areas of physics, ranging from condensed matter [19] to quantum chromodynamics [20], and its realization in cold lattice gases is attracting growing attention [21]. Schemes have been recently proposed for multicomponent lattice gases, such that the low-energy description of these systems is that of relevant quantum field theories [22-31]. The backaction of the atoms on the value of a synthetic gauge field is expected to lead to interesting physics, including statistically induced phase transitions and anyons in 1D lattices [32], and chiral solitons in Bose-Einstein condensates [33].

Periodically modulated optical lattices open interesting possibilities for the engineering of lattice gases [16-18,34-40]. In particular, periodic lattice shaking results in a modified hopping rate [34-36], which has been employed to drive the superfluid (SF) to Mott insulator (MI) transition [37], to simulate frustrated classical magnetism [38], and to create tunable gauge potentials [16]. Interestingly, a periodically modulated magnetic field may be employed in the vicinity of a Feshbach resonance to induce periodically modulated interactions, which result in a nonlinear hopping rate that depends on the occupation differences at neighboring sites [41-43].

In this Letter, we show that combining periodic interactions and Raman-assisted hopping may induce a densitydependent gauge field in 1D lattices. The created field results in a density-dependent shift of the momentum distribution that may be probed in time-of-flight (TOF) experiments. Moreover, contrary to the Peierls phase induced in shaken lattices [16], the created field cannot be gauged out and, hence, affects significantly the ground-state properties of the lattice gas, leading to gauge-induced SF to MI transitions, the emergence of MIs at vanishing interaction, and strongly modified correlations in the SF regime.

$A B$ model.-We introduce, in the following, a possible setup that creates a density-dependent Peierls phase that cannot be gauged out. We consider a tilted 1D spindependent lattice (see Fig. 1), in which atoms in state $|1\rangle(|2\rangle)$ are confined in the sublattice $A(B)$. A first pair of Raman lasers induces Raman-assisted hopping between an $A$ site and the $B$ site to its right, whereas a second pair leads to hopping between an $A$ site and the $B$ site to its left [44]. We consider that, within a period $T$, for $0<t<T / 2$, the Raman assisted coupling $A B(B A)$ is on (off) and vice versa for $T / 2<t<T$. The Hamiltonian of the system is

$$
\begin{aligned}
\hat{H}^{A B}= & -\sum_{j}\left[J_{A B}(t) \hat{b}_{2 j}^{\dagger} \hat{b}_{2 j+1}+J_{B A}(t) \hat{b}_{2 j}^{\dagger} \hat{b}_{2 j-1}+\text { H.c. }\right] \\
& +\frac{U_{A}(t)}{2} \sum_{j} \hat{n}_{2 j}\left(\hat{n}_{2 j}-1\right)+\frac{U_{B}}{2} \sum_{j} \hat{n}_{2 j+1}\left(\hat{n}_{2 j+1}-1\right),
\end{aligned}
$$

where $J_{A B}=J$ and $J_{B A}=0$ for $0<t<T / 2, J_{A B}=0$ and $J_{B A}=J$ for $T / 2<t<T$, and even (odd) site index corresponds to the $A(B)$ sublattice. We consider that the interaction of components $|1\rangle$ can be independently modulated from those of $|2\rangle$, such that $U_{A}=U_{A 0}+U_{A 1}(t)$, with $U_{A 1}(t)=U_{A 1}(t+T)$ and $\int_{t}^{t+T} d t^{\prime} U_{A 1}\left(t^{\prime}\right)=0$, whereas $U_{B}$ is constant (we consider for simplicity $U_{A 0}=U_{B} \equiv U$ [45]). As shown in Refs. [41,42], a sufficiently fast modulation of the interactions leads to an effective model with a density-dependent hopping (as discussed in the Supplemental Material [46], just modulating the interactions 
(a)

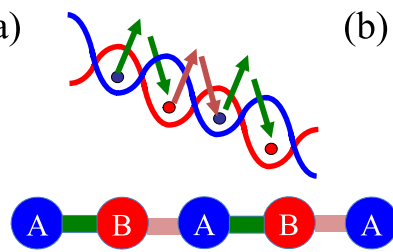

(b)

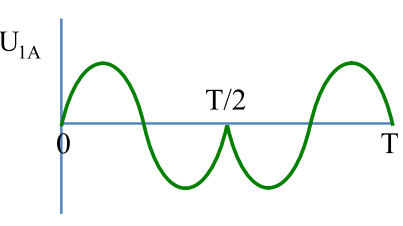

FIG. 1 (color online). Scheme of the $A B$ set up: (a) for $0<$ $t<T / 2$ Raman assisted hopping couples an $A$ site with the $B$ site at their right; for $T / 2<t<T$, it couples an $A$ site with the $B$ site at their left; (b) the $U_{A 1}(t)$ function is $\sin \left(\omega_{A B} t\right)$ for $0<t<T / 2$ and $-\sin \left(\omega_{A B} t\right)$ for $T / 2<t<T$, with $\omega_{A B}=4 \pi / T$.

in a standard Bose-Hubbard model does result in a densitydependent Peierls phase, but this phase can be gauged out [46]). For the particular case of the $A B$ model, we obtain, for a fast modulation, the effective Hamiltonian [47]

$$
\begin{aligned}
\hat{H}_{\text {eff }}^{A B}= & -\sum_{j}\left[\hat{b}_{2 j}^{\dagger} \tilde{J}_{A B}\left(\hat{n}_{2 j}\right) \hat{b}_{2 j+1}+\hat{b}_{2 j}^{\dagger} \tilde{J}_{B A}\left(\hat{n}_{2 j}\right) \hat{b}_{2 j-1}+\text { H.c. }\right] \\
& +\frac{U}{2} \sum_{j} \hat{n}_{2 j}\left(\hat{n}_{2 j}-1\right)+\frac{U}{2} \sum_{j} \hat{n}_{2 j+1}\left(\hat{n}_{2 j+1}-1\right),
\end{aligned}
$$

with $\quad \tilde{J}_{A B}\left(\hat{n}_{2 j}\right)=(J / T) \int_{0}^{T / 2} d t e^{i V(t) \hat{n}_{2 j} / \hbar}, \quad \tilde{J}_{B A}\left(\hat{n}_{2 j}\right)=$ $(J / T) \int_{0}^{T / 2} d t e^{i V(t+T / 2) \hat{n}_{2 j} / \hbar}$, and $V(t)=\int_{0}^{t} U_{A 1}\left(t^{\prime}\right) d t^{\prime}$.

For $U_{A 1}(t)=\tilde{U}_{A 1} \sin \left(\omega_{A B} t\right)$ for $0<t<T / 2$ (with $\left.\omega_{A B}=4 \pi / T\right)$, and $U_{A 1}(t)=-\tilde{U}_{A 1} \sin \left(\omega_{A B} t\right)$ for $T / 2<$ $t<T$ [see Fig. 1(b)], $\tilde{J}_{A B}\left(\hat{n}_{2 j}\right)=(J / 2) J_{0}\left(\Omega_{A B} \hat{n}_{2 j}\right) e^{i \Omega_{A B} \hat{n}_{2 j}}$, whereas $\tilde{J}_{B A}\left(\hat{n}_{2 j}\right)=\tilde{J}_{A B}\left(\hat{n}_{2 j}\right)^{*}$, with $\Omega_{A B}=\tilde{U}_{A 1} / \hbar \omega_{A B}$. For more general forms of $U_{A 1}(t)[46], \arg \left[\tilde{J}_{A B}\right]=\phi_{A B} \hat{n}_{2 j}$ and $\arg \left[\tilde{J}_{B A}\right]=\phi_{B A} \hat{n}_{2 j}$. The created Peierls phase cannot be gauged out if $\Phi \equiv \phi_{A B}-\phi_{B A} \neq 0$, crucially altering the ground-state properties.

Quasimomentum distribution.-The created Peierls phase results in a drift of the quasimomentum distribution in the SF regime. As in recent experiments on shaken lattices [16], this shift may be probed in TOF (details about experimental detection are discussed below). Figure 2(a) shows the
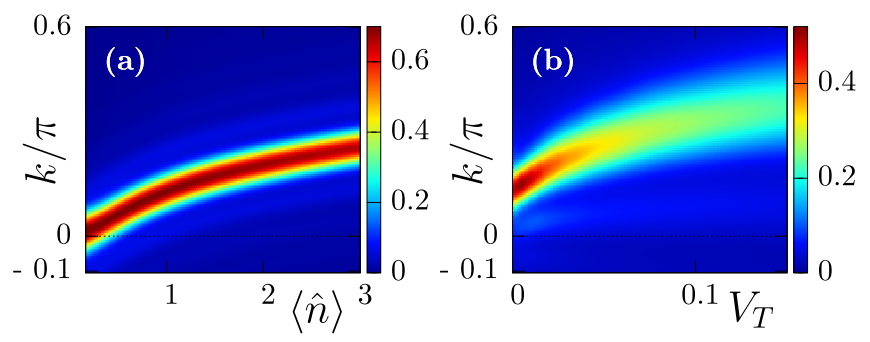

FIG. 2 (color online). (a) Ground-state quasimomentum distribution for model (2) for a homogeneous distribution in 24 sites with $\Omega_{A B}=\pi / 4, U=0.2 \mathrm{~J}$, and a density $\langle\hat{n}\rangle$; (b) same for a harmonically trapped gas as a function of $V_{T}$ (see text) for $\Omega_{A B}=\pi / 4, U=J$, and 24 particles in 24 sites. Both figures show density-matrix renormalization group (DMRG) [48] results with 500 states, and a maximal occupation per site $n_{\max }=10$.

quasimomentum distribution as a function of the average density $\langle\hat{n}\rangle$ for a homogeneous system with $\Omega_{A B}=\pi / 4$ and $U=0.2 J$. However, in contrast to shaken lattice experiments, the momentum shift is density dependent. This dependence results in a nontrivial behavior of the quasimomentum distribution in the presence of an external harmonic confinement, which may be accounted for by an additional term $V_{T} \sum_{j}(j-L / 2)^{2} \hat{n}_{j}$ in the Hamiltonian (2). As shown in Fig. 2(b), for larger $V_{T}$, the quasimomentum distribution shifts due to growing central density and broadens due to the inhomogeneous density distribution $\left\langle\hat{n}_{j}\right\rangle$.

Ground-state phase diagram.-The nongaugeable density-dependent Peierls phase and the associated broken $A B$ symmetry are crucial for the ground-state physics of the $A B$ model (see Fig. 3, in which $\mu$ is the chemical potential). MI phases at half-integer filling are induced by the $A B$ asymmetry, opening, immediately, at any finite $J$. For $\langle\hat{n}\rangle=1 / 2$ at $J / U \ll 1$, we may project on the manifold with 0 or 1 particle per site and we may identify $|0\rangle \rightarrow|\uparrow\rangle$ and $|1\rangle \rightarrow|\downarrow\rangle$, obtaining up to $\mathcal{O}\left(J^{2} / U\right)$ the effective spin- $\frac{1}{2}$ Hamiltonian $\hat{H}_{1 / 2}=$ $\hat{H}_{0}+\hat{H}_{2}$, with $\hat{H}_{0}=-J \sum_{j} \hat{S}_{j}^{+} \hat{S}_{j+1}^{-}+$H.c., and $\left(U / J^{2}\right) \hat{H}_{2}=$ $\sum_{j}\left[\hat{S}_{2 j}^{+}\left(\frac{1}{2}+\hat{S}_{2 j+1}^{z}\right) \hat{S}_{2 j+2}^{-}+\Gamma^{2} \hat{S}_{2 j-1}^{+}\left(\frac{1}{2}+\hat{S}_{2 j}^{z}\right) \hat{S}_{2 j+1}^{-}+\right.$H.c. $]-$ $\left(1+|\Gamma|^{2}\right) \sum_{j} \hat{S}_{j}^{z} \hat{S}_{j+1}^{z}$, with $\Gamma \equiv \frac{1}{2} J_{0}\left(\Omega_{A B}\right) e^{i \Phi / 2}$. Hence, the perturbative corrections result in nearest neighbor interactions and staggered correlated hopping. Following similar arguments as those employed for the treatment of the spinPeierls problem [49], one may show that the staggered correlated hopping becomes immediately relevant (in the renormalization group sense) for free hard-core particles, and hence, any $A B$-dependent $\Gamma$ opens a (band insulator) gapped phase at half-filling for $U \rightarrow \infty$ (see Supplemental Material [46] for details). A similar reasoning applies for higher half-integer fillings $\bar{n}+1 / 2$, by considering hardcore particles on top of a pseudovacuum with $\bar{n}$ particles per site. Note that the Mott boundaries depend on $\Phi$, and hence, varying $\Phi$ at constant $J / U$ results in gauge-induced phase transitions [Fig. 3(b)], similar to the statistical transitions of Ref. [32]. In particular, for $\Phi \rightarrow \pi$ one observes a strong enhancement of the MI gaps. Half-integer and integer MIs may be revealed by the appearance of density plateaus in the presence of a harmonic trap [50].

Vanishing on-site interaction.-The effect of the densitydependent hopping is particularly relevant in the regime of vanishing interaction, $U / J \rightarrow 0$. In this regime, for the usual Hubbard model, the system becomes unstable for $\mu>-J$; i.e., any filling factor becomes possible [note the bunching of curves of constant filling for $\Omega_{A B}=0$ in Fig. 3(c)]. The presence of density-dependent hopping stabilizes the system at low fillings [Fig. 3(c)]. Moreover, the $A B$ asymmetry results in a MI at half-filling even for $U / J=0$. This anomalous behavior results from the effective repulsive character of the gas even when $U=0$. This may be understood from the two-particle problem, which 

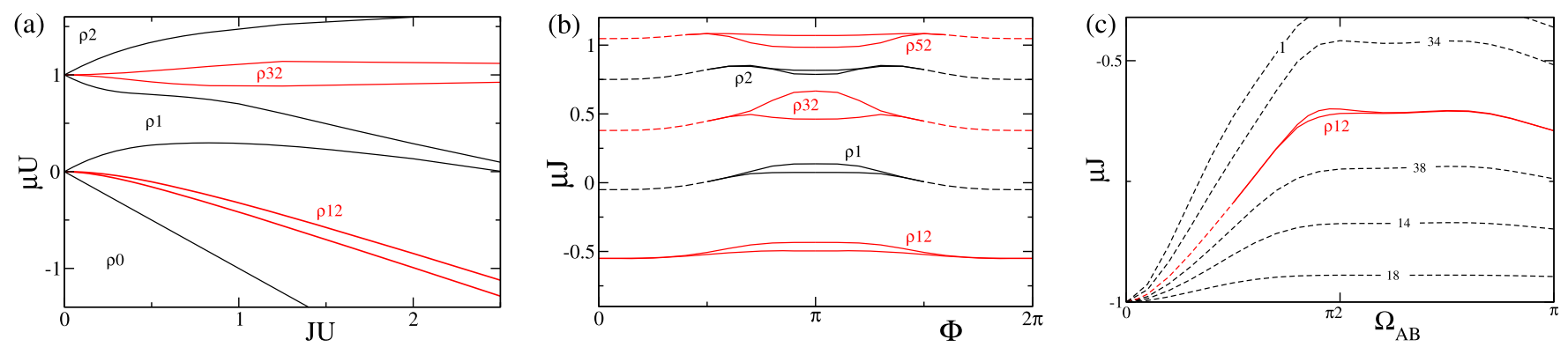

FIG. 3 (color online). Mott phases at half-integer and integer fillings of model (2). (a) MI lobes for $\Omega_{A B}=\pi / 2$, $\Phi=\pi$. (b) Varying the relative phase $\Phi$ may induce phase transitions in the ground state. Here, we choose $\Omega_{A B}=\pi / 2$ and change $\Phi[46]$ for $J / U=2$ (dashed lines indicate a closing gap). (c) Lines of constant density and MI phase at half-filling for vanishing on-site interactions $U=0$. In the DMRG-calculation system size $L$ and maximal occupation number of bosons per site $n_{\max }$ have been scaled carefully (up to $n_{\max }=12$ and $L=144$ sites) till a convergence was reached.

provides a useful description in the dilute limit [51]. The effective scattering length (in lattice spacing units) becomes of the form [46]

$$
a(U \rightarrow 0)=\frac{[3+5 \cos (\Phi)]|\Gamma|^{2}+2}{[5+3 \cos (\Phi)]|\Gamma|^{2}-2} .
$$

By comparison to a 1D Bose gas of particles with mass $m$ and contact interaction, one may extract an effective interaction strength $g=-2 /(\mathrm{am})$ [51]. The scattering length diverges for $|\Gamma| \rightarrow 1 / 2, \Phi \rightarrow 0,2 \pi$ but remains finite and negative for any other phase $\Phi$ which coincides with the observation that the $A B$-correlated hopping Hubbard model behaves as a repulsively interacting system for small filling even in the limit of $U \rightarrow 0$. Incidentally, we would like to mention that this effect may be observed, as well, for the anyon model of Ref. [32], although, in that case, the Mott plateau at half-filling is absent.

Correlation functions in the superfluid regime.The density-dependent gauge has important consequences for the correlations in the SF regime [46]. This is best understood by employing bosonization [49]: $\hat{b}_{j}^{\dagger} \rightarrow \sqrt{\rho\left(x_{j}\right)} e^{-i\left[\theta\left(x_{j}\right)-\eta x_{j}\right]}$, with $\rho(x)=\rho_{0}-(1 / \pi) \nabla \phi(x)+$ $\rho_{0} \sum_{p \neq 0} e^{i 2 p\left[\pi \rho_{0}+\phi(x)\right]}, \rho_{0}$ the average density, and $x_{j}$ the position of site $j$. The fields $\theta(x)$ and $\phi(x)$ characterize the density and phase, respectively, whereas $\eta$ is for a global gaugeable phase shift. The bosonized Hamiltonian acquires the form [46]

$$
\hat{H}=\frac{u}{2 \pi} \int d x\left[K^{-1}\left(\partial_{x} \phi\right)^{2}+K\left(\partial_{x} \theta\right)^{2}+2 \gamma\left(\partial_{x} \phi\right)\left(\partial_{x} \theta\right)\right],
$$

where $u$ is a velocity, $K$ is the Luttinger parameter, and $\gamma$ characterizes a mixing term that stems from the densitydependent Peierls phase. The decay of single particle correlations depends only on $K$ as $\left\langle\hat{b}_{i}^{\dagger} b_{j}\right\rangle \propto|i-j|^{-1 / 2 K}$ [46]. As depicted in Fig. 4, $K$ decreases with increasing $\Omega_{A B}$. This behavior can be understood already from the weak-coupling regime, in which $K$ may be determined analytically [46]

$$
K^{2}=\frac{\pi^{2} \rho_{0} \tilde{F}\left(\rho_{0}\right)}{\frac{2 U}{J}-\mathcal{R}\left(\rho_{0} \frac{d^{2} \tilde{F}}{d \rho^{2}}\left(\rho_{0}\right)+2 \frac{d \tilde{F}}{d \rho}\left(\rho_{0}\right)\right)},
$$

with $\mathcal{R}$ the real part, $\tilde{F}(\rho)=F(\rho) e^{-i \arg \left[F\left(\rho_{0}\right)\right]}$, and $F(\rho)=$ $J_{0}\left(\Omega_{A B} \rho\right) e^{i \Omega_{A B} \rho}$ for the $A B$ model [but the result may be generally applied to other forms of density-dependent tunneling, $F\left(\hat{n}_{j}\right)$ ]. Figure 4 shows that our DMRG results are in excellent agreement with Eq. (5) for small $\Omega_{A B}$, which corresponds to the weak-coupling limit. The reduction of $K$ results, on one hand, from the trivial reduction of the hopping strength $\left[J \rightarrow J \tilde{F}\left(\rho_{0}\right)\right]$, and on the other hand from a nontrivial contribution due to the density dependence (denominator of $K$ ). The latter stems from the effective repulsion discussed above. Note, in particular, that a density-dependent Peierls phase, with $|F(\rho)|=1$, as that of Ref. [32], would lead, as well, to strongly modified correlations characterized by a significant reduction of $K$ [46]. The modification of $K$ due to the density-dependent gauge may be directly probed by monitoring the form of the central momentum peak [52].

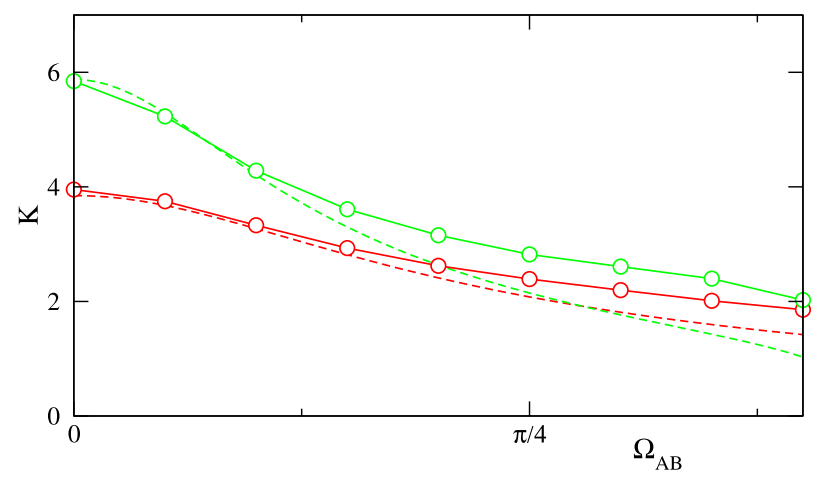

FIG. 4 (color online). Behavior of the Luttinger parameter $K$ as a function of $\Omega_{A B}$ for $U=J / 2$ for $\rho_{0}=1.75$ (upper curves) and $\rho_{0}=0.75$ (lower curves). Dashed lines indicate the analytical estimation (5) in the weakly interacting regime, whereas the circles denote our results obtained from DMRG calculations of the single-particle correlation function. 


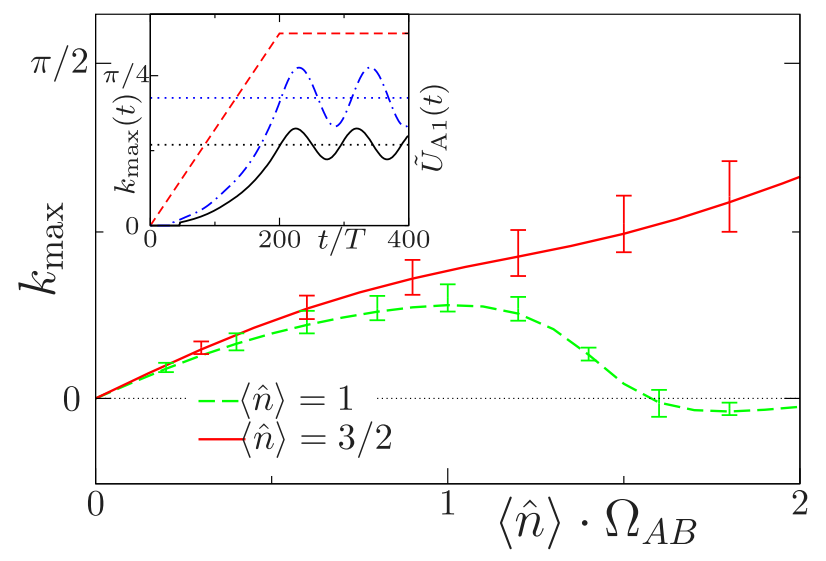

FIG. 5 (color online). Quasimomentum $k_{\max }$ at which the quasimomentum distribution of the $B$ sublattice is maximal as a function of $\Omega_{A B}\langle\hat{n}\rangle$ for $\omega=20 J$ and $U=J$. Solid (dashed) lines denote the results obtained from the effective model (2) with $\langle\hat{n}\rangle=3 / 2(1)$. The error bars denote the uncertainty (time average and standard deviation for $200<t / T<400)$ of $k_{\max }(t)$ for the case of a linear ramp of $\tilde{U}_{A 1}$ with a ramp time of $\tau=200 T$ (see text). Inset: Solid and dashed-dotted lines show $k_{\max }(t)$ for $\langle\hat{n}\rangle=3 / 2$ with $\Omega_{A B}=0.4$ and 0.8 , whereas the dotted line indicates the value of $k_{\max }$ for the effective model (2). We depict, with a dashed line, the $\operatorname{ramp} \tilde{U}_{A 1}(t)$.

Adiabatic preparation.-We have focused above on the effective model (2). As for shaken lattices [53], one may start from the ground state without modulated interactions, and adiabatically increase $\tilde{U}_{A 1}$. We have studied this preparation by means of time-evolving block decimation (TEBD) simulations [54] of the dynamics of Eq. (1) when applying a linear $\operatorname{ramp} \tilde{U}_{A 1}(t)=(t / \tau) \tilde{U}_{A 1}$ for $t<\tau$, and constant afterwards [46]. Figure 5 depicts the value $k_{\max }$ at which the momentum distribution is maximal, showing that the evolved momentum distribution is in very good agreement with that of the effective model. Note that the drift $k_{\max }$ is only linear with $\Omega_{A B}\langle\hat{n}\rangle$ for a sufficiently small value of $\Omega_{A B}\langle\hat{n}\rangle$. For larger $\Omega_{A B}\langle\hat{n}\rangle$, it presents a nontrivial density dependence, especially at low $\langle\hat{n}\rangle$, due to number fluctuations.

Detection.-Whereas the density distribution of the effective model corresponds to that measured in the laboratory frame, the measurement of the momentum distribution in TOF presents some features that differ significantly from the shaken lattice case [16]. First, since the lattice is not actually shaken, the overall momentum envelope resulting from the Fourier transform of the Wannier functions does not oscillate in time. Second, whereas the momentum distribution of the $B$ sublattice measured in TOF corresponds to that of the effective model, the distribution of the $A$ sublattice just coincides with that of the effective model (and, also, with that of the sublattice $B$ ) when $V(t)=0$. For intermediate times, the phase appearing in the conversion between both reference frames leads to a broadening, and eventual blurring, of the TOF peaks [46].
Outlook.-Periodic interactions combined with Ramanassisted hopping may create a density-dependent Peierls phase that results in nontrivial ground-state properties, characterized by a density-dependent momentum distribution, gauge-induced SF to MI transitions, the stabilization of the Hubbard model at vanishing interactions, and modified correlations in the SF phase. Although our discussion has focused on the specific case of the $A B$ model, these peculiar properties are general for all models with a density-dependent Peierls phase [55] (in the Supplemental Material [46] we comment on the case of the anyonic model of Ref. [32]).

The $A B$ model may be extended to create a densitydependent gauge field in a square lattice, in which each row is an exact copy of the $A B$ lattice as that discussed above, and rows are coupled by direct (not Raman-assisted) hops. Tilting the lattice leads to a row-dependent $\langle\hat{n}\rangle$ and, hence, to a different Peierls phase at each row when modulating the interactions. In this way, a finite flux may be produced in each plaquette, proportional to the density difference between neighboring rows. As a result, density dependent synthetic magnetic fields may be created, opening interesting possibilities that deserve further investigation.

We thank C. de Morais Smith, M. Di Liberto, M. Dalmonte, A. Eckardt, and U. Schneider for enlightening discussions. We acknowledge support by the Cluster of Excellence Centre for Quantum Engineering and SpaceTime Research, the DFG Research Training Group 1729, and the Singapore University of Technology and Design start-up Grant No. SRG-EPD-2012-045.

[1] J. Dalibard, F. Gerbier, G. Juzeliunas, and P. Öhberg, Rev. Mod. Phys. 83, 1523 (2011).

[2] N. Goldman, G. Juzeliunas, P. Öhberg, and I. B. Spielman, arXiv: 1308.6533

[3] Y. J. Lin, R. Compton, A. Perry, W. Phillips, J. Porto, and I. Spielman, Phys. Rev. Lett. 102, 130401 (2009).

[4] Y. J. Lin, R. L. Compton, K. Jiménez-García, J. V. Porto, and I. B. Spielman, Nature (London) 462, 628 (2009).

[5] Y. J. Lin, R. L. Compton, K. Jiménez-García, W. D. Phillips, J. V. Porto, and I. B. Spielman, Nat. Phys. 7, 531 (2011).

[6] Y. J. Lin, K. Jiménez-García, and I. B. Spielman, Nat. Phys. 471, 83 (2011).

[7] P. Wang, Zeng-Qiang Yu, Z. Fu, J. Miao, L. Huang, S. Chai, H. Zhai, and J. Zhang, Phys. Rev. Lett. 109, 095301 (2012).

[8] L. W. Cheuk, A. T. Sommer, Z. Hadzibabic, T. Yefsah, W. S. Bakr, and M. W. Zwierlein, Phys. Rev. Lett. 109, 095302 (2012).

[9] J. Y. Zhang et al., Phys. Rev. Lett. 109, 115301 (2012).

[10] Z. Fu, L. Huang, Z. Meng, P. Wang, L. Zhang, S. Zhang, H. Zhai, P. Zhang, and J. Zhang Nat. Phys. 10, 110 (2014).

[11] L. Zhang, Jin-Yi Zhang, Si-Cong Ji, Zhi-Dong Du, H. Zhai, Y. Deng, S. Chen, P. Zhang, and Jian-Wei Pan, Phys. Rev. A 87, 011601(R) (2013). 
[12] C. Qu, C. Hamner, M. Gong, C. Zhang, and P. Engels, Phys. Rev. A 88, 021604(R) (2013).

[13] L. J. LeBlanc, M. C. Beeler, K. Jiménez-García, A. R. Perry, S. Sugawa, R. A Williams, and I. B. Spielman, New J. Phys. 15, 073011(2013).

[14] M. Aidelsburger, M. Atala, S. Nascimbène, S. Trotzky, Y.-A. Chen, and I. Bloch Phys. Rev. Lett. 107, 255301 (2011).

[15] K. Jiménez-García, L. J. LeBlanc, R. A. Williams, M. C. Beeler, A. R. Perry, and I. B. Spielman, Phys. Rev. Lett. 108, 225303 (2012).

[16] J. Struck, C. Ölschläger, M. Weinberg, P. Hauke, J. Simonet, A. EckardtM. Lewenstein, K. Sengstock, and P. Windpassinger, Phys. Rev. Lett. 108, 225304 (2012).

[17] M. Aidelsburger, M. Atala, M. Lohse, J. T. Barreiro, B. Paredes, and I. Bloch, Phys. Rev. Lett. 111, 185301 (2013).

[18] H. Miyake, G. A. Siviloglou, C. J. Kennedy, W. C. Burton, and W. Ketterle, Phys. Rev. Lett. 111, 185302 (2013).

[19] M. Levin and X. G. Wen, Rev. Mod. Phys. 77, 871 (2005).

[20] J. Kogut, Rev. Mod. Phys. 55, 775 (1983).

[21] U. J. Wiese, Ann. Phys. (N.Y.) 525, 777 (2013).

[22] J. I. Cirac, P. Maraner, and J. K. Pachos, Phys. Rev. Lett. 105, 190403 (2010).

[23] E. Zohar and B. Reznik, Phys. Rev. Lett. 107, 275301 (2011).

[24] E. Kapit and E. Mueller, Phys. Rev. A 83, 033625 (2011).

[25] E. Zohar, J. I. Cirac, and B. Reznik, Phys. Rev. Lett. 109, 125302 (2012).

[26] D. Banerjee, M. Dalmonte, M. Müller, E. Rico, P. Stebler, U.-J. Wiese, and P. Zoller, Phys. Rev. Lett. 109, 175302 (2012).

[27] L. Tagliacozzo, A. Celi, P. Orland, M. W. Mitchell, and M. Lewenstein, Nat. Commun. 4, 2615 (2013).

[28] E. Zohar, J. I. Cirac, and B. Reznik, Phys. Rev. Lett. 110, 055302 (2013).

[29] D. Banerjee, M. Bögli, M. Dalmonte, E. Rico, P. Stebler, U.-J. Wiese, and P. Zoller, Phys. Rev. Lett. 110, 125303 (2013).

[30] E. Zohar, J. I. Cirac, and B. Reznik, Phys. Rev. Lett. 110, 125304 (2013).

[31] L. Tagliacozzo, A. Celi, P. Orland, M. W. Mitchell, and M. Lewenstein, Nat. Commun. 4, 2615 (2013).

[32] T. Keilmann, S. Lanzmich, I. McCulloch, and M. Roncaglia, Nat. Commun. 2, 361 (2011).

[33] M. J. Edmonds, M. Valiente, G. Juzeliunas, L. Santos, and P. Ohberg, Phys. Rev. Lett. 110, 085301 (2013).

[34] A. Eckardt, C. Weiss, and M. Holthaus, Phys. Rev. Lett. 95, 260404 (2005).

[35] H. Lignier, C. Sias, D. Ciampini, Y. Singh, A. Zenesini, O. Morsch, and E. Arimondo, Phys. Rev. Lett. 99, 220403 (2007).

[36] E. Kierig, U. Schnorrberger, A. Schietinger, J. Tomkovic, and M. K. Oberthaler, Phys. Rev. Lett. 100, 190405 (2008).

[37] A. Zenesini, H. Lignier, D. Ciampini, O. Morsch, and E. Arimondo, Phys. Rev. Lett. 102, 100403 (2009).

[38] J. Struck, C. Olschlager, R. Le Targat, P. Soltan-Panahi, A. Eckardt, M. Lewenstein, P. Windpassinger, and K. Sengstock, Science 333, 996 (2011).

[39] Y.-A. Chen, S. Nascimbène, M. Aidelsburger, M. Atala, S. Trotzky, and I. Bloch, Phys. Rev. Lett. 107, 210405 (2011).
[40] R. Ma, M. E. Tai, P M. Preiss, W. S. Bakr, J. Simon, and M. Greiner, Phys. Rev. Lett. 107, 095301 (2011).

[41] J. Gong, L. Morales-Molina, and P. Hänggi, Phys. Rev. Lett. 103, 133002 (2009).

[42] Á. Rapp, X. Deng, and L. Santos, Phys. Rev. Lett. 109, 203005 (2012).

[43] M. Di Liberto, C. E. Creffield, G. I. Japaridze, and C. Morais Smith, Phys. Rev. A 89, 013624 (2014).

[44] The laser arrangement is basically the same as that of [D. Jaksch and P. Zoller, New J. Phys. 5, 56 (2003)] proposed for the creation of a synthetic (static) magnetic field. However, here, we do not demand a spatial dependence of the Rabi frequencies, and the $\boldsymbol{A} \boldsymbol{B}$ and $\boldsymbol{B} \boldsymbol{A}$ lasers are switched on and off. The tilting must be sufficiently large to be resolved by the two different Raman pairs. The tilting must also be larger than the Raman-induced hopping rate and the interaction energy.Note, also, that the tilting should be chosen avoiding photon-assisted resonances [C. Sias, H. Lignier, Y. Singh, A. Zenesini, D. Ciampini, O. Morsch, and E. Arimondo, Phys. Rev. Lett. 100, 040404 (2008)], which could result in a significant $\boldsymbol{B} \boldsymbol{A}$ hopping even during the $\boldsymbol{A} \boldsymbol{B}$ pulses.

[45] An even more intriguing phase space could emerge from assuming $U_{A 0} \neq U_{B}$, however, this goes beyond the scope of this current Letter.

[46] See Supplemental Material at http://link.aps.org/ supplemental/10.1103/PhysRevLett.113.215303 for details on periodically modulated interactions, the strongly interacting limit, the calculation of the scattering length for vanishing interaction, time of flight imaging, correlation functions in the SF regime, and the numerical simulations.

[47] The $A B$ model resembles the anyon model of Ref. [32], in which the intersite hopping depends on the occupation of the left site. The model of Ref. [32] requires twice as many Raman lasers as the maximal occupation per site, and on-site interactions larger than the laser linewidth. The $A B$ model works with only one laser pair, and for interaction shifts smaller than the laser linewidth (for $\langle\hat{n}\rangle=5$ and $U=0.2 J$, the linewidth required must be larger than $U\langle\hat{n}\rangle=J$; for $J$ of the order of tens of $\mathrm{Hz}$, this is a realistic assumption for typical linewidths [18]). The $A B$ model may be recast as an anyon model without Peierls phase by defining $\hat{a}_{2 j}=e^{i \Omega_{A B} \sum_{l<j} \hat{n}_{2 l}} \hat{b}_{2 j}$, and $\hat{a}_{2 j+1}=e^{i \Omega_{A B} \sum_{l \leq j} \hat{n}_{2 l}} \hat{b}_{2 j+1}$, where the $\hat{a}$ operators fulfill, for $j^{\prime}>j, e^{i \Omega_{A B}} \hat{a}_{2 j}^{\dagger} \hat{a}_{2 j^{\prime}}=\hat{a}_{2 j^{\prime}} \hat{a}_{2 j}^{\dagger}$, $e^{i \Omega_{A B}} \hat{a}_{2 j}^{\dagger} a_{2 j^{\prime}+1}=\hat{a}_{2 j^{\prime}+1} \hat{a}_{2 j}^{\dagger}, \hat{a}_{2 j+1}^{\dagger} \hat{a}_{2 j^{\prime}+1}=\hat{a}_{2 j^{\prime}+1} \hat{a}_{2 j+1}^{\dagger}$.

[48] U. Schollwöck, Ann. Phys. (N.Y.) 326, 96 (2011).

[49] Th. Giamarchi, Quantum Physics in One Dimension, (Oxford University Press, New York, 2004).

[50] J. Sherson, C. Weitenberg, M. Endres, M. Cheneau, I. Bloch, and S. Kuhr, Nature (London) 467, 68 (2010).

[51] A. K. Kolezhuk, F. Heidrich-Meisner, S. Greschner, and T. Vekua, Phys. Rev B 85, 064420 (2012).

[52] B. Paredes, A. Widera, V. Murg, O. Mandel, S. Fölling, I. Cirac, G. V. Shlyapnikov, T. W. Hänsch, and I. Bloch, Nature (London) 429, 277 (2004).

[53] D. Poletti and C. Kollath, Phys. Rev. A 84, 013615 (2011).

[54] A. J. Daley, C. Kollath, U. Schollwöck, and G. Vidal, J. Stat. Mech. (2004) P04005.

[55] Only the half-integer MI and the MI at vanishing interactions demand, necessarily, the $A B$ asymmetry specific to the $A B$ model. 